Questions vives

\section{Questions Vives}

Recherches en éducation

$\mathbf{N}^{\circ} 32 \mid 2019$

L'accueil et la formation linguistique des migrants. Quelles pratiques aujourd'hui?

\title{
Des données de terrain à la conception d'outils de médiation de l'information : Élaborer une démarche d'information adaptée à destination des MNA
}

From field data to mediation tools: developing an approach of adapted information for Unacompanied Minors

Isabelle Estève

\section{(2) OpenEdition}

\section{Édition électronique}

URL : https://journals.openedition.org/questionsvives/4201

DOI : 10.4000/questionsvives.4201

ISSN : $1775-433 \mathrm{X}$

Éditeur

Université Aix-Marseille (AMU)

\section{Édition imprimée}

Date de publication : 30 décembre 2019

ISBN : 978-2-912643-56-8

ISSN : 1635-4079

\section{Référence électronique}

Isabelle Estève, «Des données de terrain à la conception d'outils de médiation de l'information :

Élaborer une démarche d'information adaptée à destination des MNA », Questions Vives [En ligne], N 32 | 2019, mis en ligne le 30 octobre 2020, consulté le 27 août 2021. URL : http://

journals.openedition.org/questionsvives/4201 ; DOI : https://doi.org/10.4000/questionsvives.4201

Ce document a été généré automatiquement le 27 août 2021.

\section{cc)}

Questions Vives est mis à disposition selon les termes de la licence Creative Commons Attribution -

Pas d'Utilisation Commerciale - Pas de Modification 4.0 International. 


\title{
Des données de terrain à la conception d'outils de médiation de l'information : Élaborer une démarche d'information adaptée à destination des MNA
}

\author{
From field data to mediation tools: developing an approach of adapted \\ information for Unacompanied Minors
}

Isabelle Estève

\section{Introduction}

Nous rendrons compte dans cet article de la démarche de conception des outils de médiation que nous avons élaborés dans le cadre du projet Médiation de l'information pour les mineurs non accompagnés (MIMNA). Ce projet vise à répondre aux besoins informationnels des mineurs non accompagnés (MNA) - adolescents migrants de moins de 18 ans, isolés sur le territoire français, qui sont accueillis en France par les dispositifs de protection de l'enfance. Il tente ainsi d'apporter une réponse à un défi qui nous semble central dans les mesures de protection de ce public mineur en situation de vulnérabilité informationnelle : optimiser le soutien social informatif à destination des MNA pour permettre une meilleure appropriation de leur environnement en France (droits, procédures administratives, codes socio-culturels, fonctionnement de l'environnement institutionnel, rôle et fonction des professionnels, etc.) et réduire leur insécurité informationnelle. Le livret d'accueil et son guide d'utilisation visent à outiller les professionnels pour qu'ils soient mieux à même de répondre aux besoins informationnels des MNA dès l'accueil d'urgence, dans le cadre de la phase règlementaire de mise à l'abri. 
2 Il s'agira de rendre compte dans cet article de la démarche de recherche-action interdisciplinaire ou plus justement transdisciplinaire, en ceci qu'elle inclut les professionnels de terrain comme partie prenante de l'équipe de recherche, au même titre que les chercheurs en psychologie interculturelle et en (socio)linguistique appliquée à la médiation, impliqués dans le projet. La démarche de recherche dans lequel s'inscrit le projet MIMNA ne consiste

pas simplement [à] appliquer la recherche à un projet sociétal, mais [à] s'inscrire dans un processus au sein duquel il s'instaure un échange durable et constructif entre recherche et société, qui sont alors interconnectés et répondent à leurs besoins mutuels (Mazur-Palandre \& Colón de Carvajal, 2019, p. 322).

Entrecroiser l'expertise des professionnels travaillant auprès des MNA, et une approche sociolinguistique des problématiques de médiation a permis de formaliser des principes et des stratégies de médiation, sur la base des données de terrains, afin d'élaborer des outils de médiation dans une démarche d'information effectivement adaptée aux MNA. Nous entendons par information adaptée le fait qu'elle permette aux MNA, non seulement d'activer du sens mais de s'approprier l'information et de construire des connaissances qui sont signifiantes pour eux.

4 Nous rendrons compte dans un premier temps de la manière dont cette rechercheaction s'est tissée autour d'une urgence de terrain liée à l'accueil et l'accompagnement des MNA par les services de protection de l'enfance, en croisant les regards: des professionnels accueillant ces publics dans un centre d'accueil d'urgence, d'un chercheur en psychologie interculturelle appliquée aux MNA et également psychologue clinicien auprès de ces publics, et d'une sociolinguiste spécialiste des problématiques d'accessibilité en contexte de surdité et plus largement de la médiation en contexte exolingue et interculturel. Nous expliciterons dans un second temps la manière dont la démarche d'information adaptée s'est étayée grâce à un protocole de recherche structuré par une approche sociolinguistique des problématiques de médiation, entrecroisant les besoins informationnels des MNA et les besoins de transmission des professionnels. Nous aborderons la manière dont ces analyses de terrain nous ont amenée à formaliser des principes et des stratégies de médiation pour l'élaboration du livret d'accueil exclusivement visuel et de son guide d'utilisation.

\section{Regards croisés sur la médiation de l'information à destination des MNA}

\subsection{Démarche de recherche-action transdisciplinaire}

5 Le projet MIMNA s'est tissé autour de l'expertise de terrain de professionnels (chef de service, psychologue, formatrices et éducateurs) d'une structure d'accueil d'urgence des MNA (Le Charmeyran). Ces professionnels, notamment sous l'impulsion de Guillaume Coron, développaient déjà, en amont du projet, une approche interculturelle d'accueil, d'accompagnement et de scolarisation des MNA (voir pour un aperçu Coron (2018), ainsi que les bonnes pratiques du Charmeyran répertoriées par le Conseil de l'Europe (2019)). Un livret visuel, composé de schématisations visuelles, pictogrammes et une information écrite minimale avait notamment été élaboré pour accueillir les MNA lors de leur entretien d'admission. L'élaboration de ce livret faisait suite aux 
constats des professionnels de la difficulté d'accueillir les MNA non francophones ${ }^{1}$ et avait une double visée :

Dispenser de l'information au MNA (environnement institutionnel, mesures administratives et évaluatives, examens médicaux, exercice de la religion dans un environnement laïque, traites des êtres humains, etc.) ;

Collecter de l'information sur le MNA (parcours migratoire, liens familiaux, santé).

6 Ce livret a été traduit dans six langues (anglais, dari, arabe, italien, espagnol, portugais). Précisons qu'il a été élaboré dans une démarche inclusive : des focus groupe ont été organisés avec les MNA pour évaluer les ajustements informationnels nécessaires à effectuer sur la première version du livret.

Figure 1. Extraits Livret d'accueil existant au Charmeyran (LC)

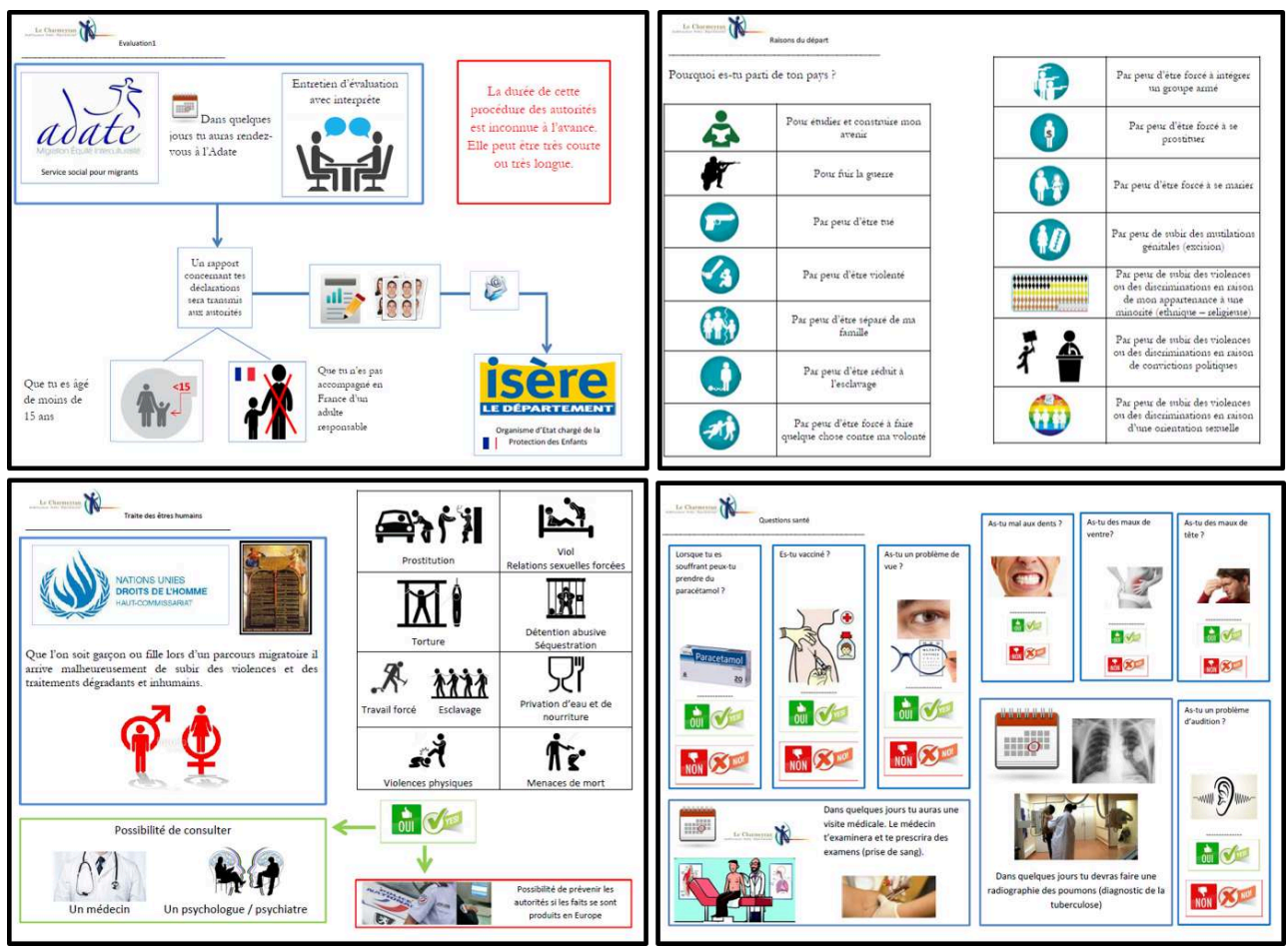

7 C'est autour de ce livret (appelé dans la suite de l'article Livret Charmeyran (LC)) que s'est structuré le protocole de recherche sur lequel nous reviendrons ultérieurement (cf. 2.2).

8 En outre, les recherches de Sydney Gaultier en psychologie interculturelle - ainsi que son expérience de psychologue clinicien auprès de ces publics - sous-tendent une hypothèse forte du projet MIMNA : l'insécurité contextuelle aggrave ou participe au maintien des troubles dont sont déjà porteurs certains jeunes (Vervliet et al., 2014). Produire un soutien social informatif adapté devrait de façon significative avoir une incidence positive sur l'insécurité informationnelle de ces jeunes et donc sur leur détresse psychologique, leur santé mentale et le sentiment de contrôle perçu.

Enfin, la démarche d'information adaptée élaborée dans le cadre du projet MIMNA s'est également largement nourrie des réflexions menées par Isabelle Estève, dans ses pratiques d'enseignement et de recherche, sur l'accessibilité à l'information/aux savoirs notamment dans le contexte de la surdité, et plus largement sur l'élaboration de dispositifs de médiation de l'information en contexte exolingue et interculturel. 
Nous entendons par dispositifs de médiation des «dispositifs propres à renforcer l'appropriation des contenus par les usagers » (Caillet \& Jacobi, 2004, cités par Liquète, 2015) nécessitant d'engager une démarche de mise en accessibilité de l'information ${ }^{2}$ qui tienne compte centralement des besoins informationnels, des bagages expérientiels, des contraintes linguistiques, culturelles, etc., des usagers. C'est en partant de ces considérations conceptuelles de la médiation de l'information que nous avons développé une démarche d'information adaptée, entendue comme le fait qu'elle permette au MNA, non seulement d'activer du sens mais de s'approprier l'information et de construire des connaissances qui sont signifiantes pour lui ${ }^{3}$.

10 C'est pour répondre à cette démarche d'information adaptée que nous avons fait le choix d'un outil non-linguistique de médiation dans une démarche de conception universelle telle que définie par le Conseil de l'Europe (2009), afin d'élaborer un support accessible à tous les MNA en prenant en compte la diversité des profils linguistiques, littéraciques, scolaires et socio-culturels des MNA, et susceptible de garantir un accès égal à l'information.

La conception universelle est une stratégie qui vise à concevoir et à composer différents produits et environnements qui soient, autant que faire se peut et de la manière la plus indépendante et naturelle possible, accessibles, compréhensibles et utilisables par tous, sans devoir recourir à des solutions nécessitant une adaptation ou une conception spéciale. (Conseil de l'Europe, 2009, p. 9)

11 Fort de ces regards transdisciplinaires posés sur la médiation de l'information à destination des MNA, le projet MIMNA s'articule autour de 2 axes principaux :

1. Favoriser l'accès à l'information par la création d'outils non-linguistiques de médiation des savoirs ;

2. Mesurer l'impact psychologique sur la détresse psychologique, la santé mentale et le sentiment de contrôle perçu.

12 Il sera davantage question de l'axe 1 dans cet article. L'axe 2 constitue une phase ultérieure du projet.

\subsection{Constats de départ}

13 Le projet MIMNA part de deux constats de terrain. Les MNA sont en situation de vulnérabilité informationnelle quand ils sont accueillis dans les centres d'accueil d'urgence. Les pratiques informationnelles des professionnels qui les accueillent sont souvent inadaptées voire inexistantes, ce qui a pour effet de renforcer la vulnérabilité informationnelle de ces publics.

\subsubsection{Vulnérabilité informationnelle des MNA ${ }^{4}$}

14 Compte tenu de leur âge ${ }^{5}$, de leur pays d'origine ${ }^{6}$, de leurs compétences linguistiques en français ${ }^{7}$, en littératie ${ }^{8}$, de leur bagage scolaire ${ }^{9}$, de leurs expériences socioculturelles, du manque de connaissances ou de connaissances erronées sur le contexte socioculturel français ${ }^{10}$, les MNA présentent une vulnérabilité informationnelle importante, tant d'un point de vue linguistique que cognitif, dans la compréhension et l'appropriation de leur nouvel environnement socio-culturel avec lequel ils ne sont pas familiers. Leur vulnérabilité informationnelle n'est pas seulement marquée par un écart entre l'environnement socio-culturel du pays d'accueil et celui de leur pays 
d'origine, mais plus largement par l'interculturalité dans l'acception large qu'en propose Marandon (2003, p. 266) :

Il y a [...] situation interculturelle dès que les personnes ou les groupes en présence ne partagent pas les mêmes univers de significations et les mêmes formes d'expression de ces significations, ces écarts pouvant faire obstacle à la communication. [...] [Ces situations de] rupture culturelle [sont] liées à divers types d'appartenance (ethnie, nation, région, religion, genre, génération, groupe social, organisationnel, occupationnel, en particulier).

\subsubsection{Des pratiques informationnelles inadaptées}

15 Par ailleurs, comme le soulignent de nombreux groupes de travail, nationaux (ANESM, 2017) et internationaux (Conseil de l'Europe, 2018), et comme nous avons pu également en rendre compte dans notre enquête de terrain auprès des MNA (Estève, Coron \& Gaultier, à paraître 2020), les pratiques informationnelles à destination des MNA sont fréquemment inadaptées voire inexistantes. Les professionnels sont souvent démunis face à l'incompréhension des MNA, en situation de "handicap partagé ${ }^{11}$ : chacun étant dans l'impossibilité de comprendre l'autre et de se faire comprendre par l'autre. Rejeter l'incompétence sur les MNA («ils ne comprennent rien», «ils parlent mal français ", « ils ne savent pas communiquer ») (Vetier, 2016) est une stratégie courante des professionnels pour se dédouaner de leur responsabilité informationnelle, ce qui génère en conséquence des pratiques informationnelles partielles voire inexistantes. Comme nous avons pu le montrer ailleurs (Estève, Coron \& Gaultier, à paraitre 2020), les représentations des professionnels sur les besoins informatifs du jeune dans ce contexte d'urgence conditionnent des impasses informationnelles nombreuses: l'information "non rentable" (dont le coût informationnel est trop important au regard du bénéfice projeté pour le jeune), l'information «non vitale» (qui ne fait pas partie des premières nécessités, attribuées aux besoins vitaux), et l'information « pour plus tard» (donnée au compte-goutte seulement si l'éducateur le juge utile). Par ailleurs, le recours aux logiques d'accessibilité inadaptées est sous-tendu par un certain nombre d'impensés concernant les besoins effectifs des MNA et les problématiques d'accès à l'information de ce public, dont nous rendrons compte plus loin dans l'article (cf. 2.1).

\subsubsection{Répondre aux besoins informationnels : une mesure centrale de protection des MNA}

16 La vulnérabilité informationnelle des MNA est ainsi peu prise en compte sur le terrain, voire renforcée par les pratiques informationnelles peu efficientes, et est par ailleurs peu renseignée dans la littérature. Ainsi, à leur arrivée dans les services de protection de l'enfance, les MNA peuvent rester plusieurs semaines, voire plusieurs mois durant, dans une incompréhension parfois totale des conditions d'accueil, de leur environnement (socio-culturel, institutionnel), des démarches d'évaluation qui affectent leur futur en France, des mesures qui sont décidées pour eux, de leurs droits. Précisons par ailleurs que la réalité de la temporalité des besoins informationnels des MNA à leur arrivée n'est souvent pas en adéquation avec la réalité des possibilités de recours à un interprète (nombre, disponibilité, langues, coûts) et à l'urgence informationnelle qui, à notre sens, relèvent de la responsabilité des professionnels. 
17 Or, compte tenu de leur vulnérabilité informationnelle, linguistique, interculturelle, psychologique et procédurale (Estève, Coron \& Gaultier, à paraître 2020), répondre aux besoins informationnels des MNA devrait être une mesure centrale de protection de ces publics. Ainsi, outiller les professionnels des services de protection de l'enfance pour qu'ils soient à même, et ce dès leur accueil, de dispenser une information effectivement adaptée aux MNA, d'étayer leurs capacités d'empowerment ${ }^{12}$, leur pouvoir d'agir sur leur environnement, est un des défis centraux auquel se propose de répondre le projet MIMNA.

\section{Pour une information effectivement adaptée au MNA : renseigner les problématiques de médiation}

Fort de ces constats de départ, le projet MIMNA entre en résonnance avec les recommandations nationales (ANESM, 2017) et européennes (Conseil de l'Europe, 2018) : l'accès à une information adaptée devrait constituer un enjeu prioritaire des mesures de protection, d'accueil et d'accompagnement des MNA.

On entend par « informations adaptées aux enfants » des informations adaptées à l'âge, au degré de maturité, à la langue, au sexe et à la culture de l'enfant. Celui qui fournit l'information devra donc l'adapter et adopter une communication plus ou moins complexe en fonction de la situation de chaque enfant et de son âge. Ces éléments devront être pris en compte cumulativement. Toute information peut [donc] être transformée en information adaptée aux enfants par celui qui la fournit, et en particulier les informations génériques sur les droits et les informations destinées aux jeunes. (Conseil de l'Europe, 2018, p. 16).

Or ces recommandations n'ont donné lieu qu'à peu d'applications pratiques sur le terrain. Les principes d'une démarche d'information adaptée permettant de répondre de manière effective aux besoins informationnels des MNA restaient donc à développer. Dans une démarche de mise en accessibilité de l'information, telle que nous l'avons définie plus haut (cf. 1.1), nous avons ainsi mis en place un protocole de recherche qui nous permettait de croiser les besoins informationnels des MNA et les besoins de transmissions des professionnels, et de pouvoir renseigner les problématiques de médiation liées à la transmission/réception de l'information/des savoirs. Bien que ces problématiques soient par ailleurs assez bien conscientisées par les professionnels de terrain partie prenante du projet, elles n'étaient pas nécessairement formalisées dans leur globalité. Les analyses de terrain ont donc constitué une matière réflexive, indispensable à la conceptualisation des outils de médiation permettant d'appréhender les problématiques d'accès à l'information des MNA peu renseignés, tant a) au niveau linguistique - compétence peu développée dans la langue du pays d'accueil et/ou dans la langue utilisée pour la traduction -, b) interculturel - rupture dans les univers de signification (Marandon, 2003) - c) qu'interactionnel - incompréhension/malentendu dû.e aux " pré-discours »13 (savoirs, croyances, pratiques) non partagés (Paveau, 2006).

\subsection{Renseigner les supports informationnels existants}

En l'absence d'état de l'art dans la littérature des pratiques informationnelles existantes à destination des MNA, une recherche systématique sur les sites internet des organismes dédiés à la migration dans les différents pays européens nous a permis 
d'identifier 38 supports d'information européens destinés aux enfants/adolescents migrants.

Une analyse de ces supports nous a permis d'identifier trois utopies qui fondent les logiques d'accessibilité à destination de ce public, que nous pourrions résumer de la façon suivante (Estève, Coron \& Gaultier, à paraître 2020) :

1. L'utopie de la transparence de l'écrit. Toute information donnée par écrit serait accessible. L'écrit, en tant que trace, permettrait de contourner les problématiques d'incompréhension à l'oral dans la langue du pays d'accueil dont les compétences sont inégalement partagées.

2. L'utopie de la traduction. La traduction serait automatiquement garante de l'accès au sens : toute information traduite serait accessible. La traduction, permettrait de contourner les problématiques d'incompréhension dans la langue du pays d'accueil, en transposant l'information dans une langue "davantage maîtrisée"14.

3. L'utopie de l'universalité du visuel. Toute information illustrée permettrait l'accès direct au sens. Le dessin (et plus largement le visuel: pictogrammes, photos, etc...) serait une ressource universelle qui permettrait de dépasser les barrières de communication liées aux langues.

Or, et comme nous l'avons précisé plus haut, à leur arrivée, les MNA ont pour la majorité d'entre eux une faible compétence linguistique en français, un bagage en littératie peu développé et sont en situation interculturelle au sens où nous l'avons développé plus haut (cf. 1.2.1). Ainsi, les pratiques informationnelles sous-tendues par ces trois utopies sont peu efficientes, voire inadaptées: le recours à des supports majoritairement écrits, à la traduction dans une langue qui n'est pas nécessairement leur langue première et qui plus est non maîtrisée à l'écrit, et à des contenus illustrés qui ne font pas sens dans leurs bagages expérientiels et/ou culturels (Estève, Coron \& Gaultier, à paraître 2020).

\subsection{Appréhender les problématiques linguistiques, interculturelles et interactionnelles ${ }^{15}$}

Les trois logiques d'accessibilité que nous avons identifiées étaient également, dans une certaine mesure, celles qui sous-tendaient le livret existant au Charmeyran (LC). Ainsi, pour mieux appréhender les problématiques d'accessibilité linguistiques, interculturelles et interactionnelles, nous avons recueilli des données de terrain autour du LC, par les MNA et les professionnels.

Accéder aux stratégies de médiation existantes chez les professionnels et aux problématiques d'accès à l'information des MNA a nécessité la mise en place d'un protocole de recherche expérimental ajusté aux réalités de terrain rendant difficile l'observation directe. D'une part, les difficultés à accéder aux entretiens d'admission, pour des raisons éthiques, psychologiques et procédurales font de ce temps d'interaction un temps de vulnérabilité trop important pour le MNA pour qu'il puisse être filmé. Et d'autre part, l'analyse multimodale des interactions pour accéder aux stratégies de médiation dans leurs réalités linguistiques et non-linguistiques nécessite un dispositif audio-visuel de captation considéré comme trop invasif dans les interactions du quotidien pour les professionnels comme pour les MNA. 


\subsubsection{Quelles problématiques d'accessibilité face aux supports existants?} ici de façon synthétique. D'une part, les représentations visuelles utilisées dans le LC (pictogrammes, schématisation, symboles, codes couleurs, éléments linguistiques) ne font sens qu'en regard de conventions et de stéréotypes socio-culturels (Bordon, 2004 ; Tourneux, 1993 ; Eco, 1970) français, avec lesquels les MNA ne sont pas familiers et qui n'ont pas de résonnance dans les connaissances préalables des MNA. Les MNA les plus faiblement littéraciés se focalisent sur les éléments visuels qui ont un sens immédiat dans leur bagage expérientiel et qui sont les moins codifiés, ce qui rend très partiel l'accès au sens. D'autre part, le support restant linguistico-centré et illustré ponctuellement par du visuel, l'accès au texte conditionne l'accès aux informations clefs de chacune des planches. Toutefois, l'accès à l'écrit et au français ne garantit pas à lui seul l'accès au sens, et ne permet pas de dépasser les problématiques interprétatives : certains mots/concepts ne renvoient à aucun sens dans les univers de signification de ces jeunes et les représentations visuelles parfois peu explicites du LC ne permettent pas de faire sens. Leur accès au sens oscille ainsi entre incompréhension et malentendus (Laforest \& Vincent, 1999) ${ }^{16}$ : entre incompréhension totale et hypothèses sur-interprétatives influencées par les pré-discours (savoirs, croyances, pratiques), et notamment ceux qui circulent entre les MNA pour tenter de combler le flou informationnel.

Ainsi, face à ces supports linguistico-centrés et ethno-centrés, l'ensemble des codes socio-langagiers, plurisémiotiques et multimodaux, culturellement déterminés pour construire du sens constituent des obstacles pour l'accès au sens des MNA. Les ruptures interculturelles (Marandon, 2003), en tant que divergence des codes de représentations, des formes de signification et des univers de signification non partagés, concernent plus largement alors l'ensemble des ressources informationnelles, linguistiques et nonlinguistiques (pictogrammes, symboles, schématisation, écrit, etc.), auxquelles les MNA sont confrontés dans leur nouvel environnement socio-culturel.

Ces analyses de terrains nous ont permis d'identifier les écueils à éviter et de formaliser des principes de représentation visuels qui puissent faire sens pour le MNA, en dehors des conventions et stéréotypes socio-culturels français (cf. 3.2.2).

\subsubsection{Problématiques interactionnelles et stratégies de médiation des professionnels}

Les analyses des entretiens de reformulation soulignant les limites du support comme vecteur de sens autonome, même pour ceux qui ont un bagage en littératie avancé, nous ont invités à renseigner la manière dont le sens se co-construit, en, dans et par l'interaction (Kerbrat-Orrechioni, 2005), entre les professionnels et les MNA autour du LC. 
30 Afin d'être davantage en prise avec les stratégies de médiation effectives des professionnels, six entretiens de simulation ont été filmés visant à reproduire la situation d'entretien d'admission. Les entretiens d'admission sont effectués de façon systématique à l'arrivée du MNA dans l'établissement et sont effectués par les chefs de service à l'appui du livret d'accueil. Les entretiens de simulation ont permis d'analyser les stratégies de médiation des professionnels autour du LC, tant d'un point de vue langagier (utilisation des ressources multimodales: parole, gestes, représentations visuelles), que d'un point de vue interactionnel (stratégies de régulation de l'intercompréhension: identification de l'incompréhension/du malentendu et stratégies de résolution).

31 Les interactions entre professionnels et MNA sont assez révélatrices des difficultés liées à la co-construction du sens autour du livret: des notions abstraites non représentées dans le livret ; des représentations visuelles peu explicites; des difficultés pour accéder au sens des notions clefs qui ne font pas partie des univers de signification des jeunes, de leur expérience, parfois contraire à leur croyance. L'incompréhension ici n'est pas linguistique mais touche aux fondements mêmes de la question interculturelle: la confrontation des univers de signification où émergent les impensés de part et d'autre, et les pré-discours (Paveau, 2006) (croyances, représentations socio-culturelles et savoirs sur le monde) non partagés entre le professionnel et le MNA. Ces ruptures interculturelles si elles ne sont pas anticipées, prises en compte, peuvent générer non seulement des obstacles à la communication mais aussi altérer, pour le MNA, l'accès à l'exercice de ses droits fondamentaux. Ces entretiens montrent également la richesse des stratégies de médiation multimodales développées par les professionnels autour du livret, pour identifier, anticiper et réguler l'intercompréhension.

32 Ces analyses ont permis de nourrir à la fois l'ajustement des planches du livret pour intégrer des représentations visuelles permettant de renforcer les stratégies de médiation déjà développées par les professionnels pour tenter de transmettre une information signifiante pour le MNA, en faisant écho à son bagage expérientiel/ culturel. Par ailleurs, les pratiques d'utilisation multimodale du livret par les professionnels ont été formalisées sous la forme de stratégies de médiation qui figurent dans le guide d'utilisation (cf. 3.2.3).

\subsubsection{Formalisation des problématiques de médiation}

L'analyse des entretiens de reformulation et de simulation nous a ainsi permis de poser un autre regard sur les problématiques linguistiques, interculturelles, interactionnelles en jeu dans l'accès à l'information de ce public et qui nécessitent d'être prises en compte dans une démarche de mise en accessibilité de l'information.

\section{Problématiques linguistiques}

34 La langue principale de l'interaction (le français) est inégalement maitrisée par les professionnels et les MNA. L'écrit (en français ou dans une autre langue) ne fait pas exception, compte tenu du faible bagage scolaire des MNA. Le recours à la traduction dans une autre langue dans laquelle on ne peut évaluer le degré de maîtrise du jeune, ne permet pas de s'assurer de l'accès au sens effectif de l'information. 


\section{Problématiques interculturelles} Pourtant, malgré cette compétence, les interactions peuvent être marquées par des ruptures interculturelles (Marandon, 2003), du fait que les professionnels et les MNA ne partagent pas les mêmes univers de significations et les mêmes formes d'expressions de ces significations. Ces ruptures culturelles ne sont pas seulement en lien avec le pays d'origine des individus, mais plus largement avec l'ensemble de leurs appartenances socio-culturelles (nation, région, ethnie, religion, genre, génération, groupe social, organisationnel, occupationnel...). Ces appartenances diverses génèrent des pré-discours (Paveau, 2006) (savoirs, croyances, pratiques, connaissances sur le monde) qui agissent comme des filtres pour l'interprétation et la production du sens et peuvent faire obstacle à la communication.

\section{Problématiques interactionnelles}

Le sens n'est pas préexistant, donné à l'avance ${ }^{17}$, mais se co-construit dans et par l'interaction, par des actions de coopération, d'ajustement, de médiation pour assurer l'intercompréhension ${ }^{18}$. L'enjeu de transmission d'une information adaptée repose donc sur l'élaboration de stratégies de médiation qui permettent au MNA d'activer du sens, de s'approprier l'information et de construire des connaissances qui sont signifiantes pour lui, en tenant compte des ruptures interculturelles et des pré-discours en présence. Le professionnel doit donc être en mesure de se décentrer de ses propres références culturelles, linguistiques et sémantiques.

Précisons par ailleurs que la présence d'un interprète a souvent pour effet de déresponsabiliser les professionnels de la régulation de l'intercompréhension. Or elle n'évacue pourtant pas ces problématiques d'accès au sens : nombre de MNA nous ont rendu compte en entretien des problématiques d'intercompréhension avec l'interprète du fait d'une compétence inégale dans la langue utilisée pour la traduction, concernant enfin les problématiques interculturelles et interactionnelles : c'est toujours le discours du professionnel qui est transposé dans l'autre langue. Il revient donc au professionnel d'adapter ses stratégies de médiation pour réguler l'intercompréhension.

C'est en tenant compte de l'ensemble des problématiques linguistiques, interculturelles, interactionnelles en jeu que le livret MIMNA a été conçu, dans l'optique de définir les principes d'une information effectivement adaptée aux MNA.

\section{Formalisation des principes et des stratégies de médiation : des données aux outils de médiation}

\subsection{Démarche de conception universelle}

\subsubsection{Un support visuel et non-linguistique}

Afin de garantir l'accès à l'information de l'ensemble des MNA dès leur accueil, et de favoriser par là-même leur empowerment, quel que soit leur niveau de français, leurs langues-cultures d'origine, la présence ou non d'un interprète à leur arrivée, quels que soient leurs profils linguistiques, scolaires et culturels, nous avons adopté une démarche de "conception universelle» (Conseil de l'Europe, 2009). Cette démarche 
vise à intégrer, dès la conception, l'ensemble des besoins spécifiques pour permettre l'élaboration de supports accessibles, compréhensibles et utilisables par tous sans nécessiter d'adaptations particulières ${ }^{19}$. Pour la conception du livret MIMNA, la prise en compte des besoins des publics faiblement littéraciés nous a amenés à élaborer un outil non-linguistique, qui peut servir les besoins informationnels de tous les MNA, compte tenu de l'interculturalité qui marque leur accueil et leur accompagnement. Le livret MIMA est donc exclusivement visuel et non-linguistique pour éviter le recours à une ou des langues spécifiques non maîtrisées par tous, et qui plus est à l'écrit; ceci pour garantir un accès égal à l'information de tous à tout.

\title{
3.1.2 Garantir un accès à l'information dans l'exercice de leurs droits fondamentaux
}

Garantir un accès égal à l'information pour tous s'inscrit, d'une part, dans la perspective d'autonomiser les MNA face à l'information, en leur permettant de construire des connaissances signifiantes pour eux, qui leur permettent d'appréhender leur nouvel environnement socio-culturel et, d'autre part, d'accroitre leur pouvoir d'agir sur leur environnement (empowerment) dans l'exercice de leurs droits fondamentaux. Notre démarche de médiation pour garantir une information effectivement adaptée au MNA rejoint ainsi les objectifs inclusifs de la démarche de conception universelle, telle que définie par le Conseil de l'Europe (2009) :

\begin{abstract}
Promouvoir la pleine participation à la vie au sein de la société, en assurant l'accès et la facilité d'utilisation de tout ce qu'offre la société, notamment l'environnement bâti, les transports, les biens et les produits, l'information, les services publics, l'éducation, l'emploi et les soins de santé ; [..] mettre en œuvre la conception universelle en tant que stratégie permettant d'assurer les mêmes droits démocratiques à tous les membres de la société, y compris des personnes handicapées, sans distinction d'âge, de capacités ou d'origine culturelle. (Conseil de l'Europe, 2009, p. 7)
\end{abstract}

41 Cette démarche est également en application directe de la loi de $2002^{20}$ concernant le droit de l'usager à être partie prenante de son parcours de prise en charge, et notamment en renforçant son accès à l'information concernant ses droits dans l'environnement institutionnel.

\subsubsection{Précisions sur les objectifs informationnels}

Le livret MIMNA vise la réduction de l'insécurité informationnelle des MNA en optimisant leur soutien social informatif pour permettre une meilleure appropriation de leur environnement en France : droits fondamentaux, procédures administratives, codes socio-culturels de l'environnement institutionnel, identité et rôle des professionnels, risques et prévention de la traite des êtres humains. Précisons cependant que cet outil permet non seulement de dispenser de l'information, mais aussi d'en collecter (identification du MNA, de sa famille, de ses problèmes de santé et de ses antécédents médicaux et scolaires).

43 Le contenu suit ainsi les dernières recommandations du Conseil de l'Europe (2018) sur l'accès à une information adaptée pour les enfants en situation de migration : l'accès aux droits, les services de protection, les procédures d'évaluation de l'âge, d'immigration (durée, résultats possibles, procédures de recours), la scolarité, la santé, les informations sur le pays où ils séjournent. Concernant les procédures, il suit la 
"circulaire relative aux modalités de prise en charge des jeunes isolés étrangers: dispositif national de mise à l'abri, d'évaluation et d'orientation" dite "Circulaire Taubira » (2013).

\subsection{Optimiser l'accès à l'information : principes et stratégies de médiation}

\subsubsection{Médiation de l'information : une approche multimodale} pour les MNA, quelles que soient leurs langues-cultures d'origine et éviter, autant que faire se peut, les biais interprétatifs. Les représentations stylisées, symboliques ou métaphoriques de types pictogramme, ne faisant sens qu'au regard de conventions et 
de stéréotypes socio-culturels, ont été évitées. Nous avons privilégié des représentations explicites et réalistes qui correspondent à une scénarisation de l'information ${ }^{21}$, pouvant faire écho à l'expérience préalable ou à venir du jeune. La conception des planches a donné lieu à un travail conséquent de conceptualisation de chaque représentation visuelle sous la forme d'un cahier des charges détaillant de façon très précise chaque scène visuelle devant être représentée par l'illustrateur. Le choix des représentations s'est nourri de l'enquête de terrain menée dans le cadre de ce projet, des points d'incompréhension et de vigilance identifiés dans les entretiens de reformulation, et de l'expertise de terrain des professionnels travaillant auprès des MNA. Pour plus de précisions sur les principes de représentations visuels, nous renvoyons le lecteur à ce que nous avons développé par ailleurs (Estève, Coron \& Gaultier, à paraître, 2020 ; Estève \& Coron, à paraître 2020). Les annexes 1 et 2 donnent un aperçu des planches « Arrivée en France » et « Hébergement ».

Nous précisons que le recours à la scénarisation de l'information nécessitait sur certaines planches de pouvoir représenter un personnage utilisé tout au long du livret qui puisse référer au jeune. Nous avons fait le choix de tenir compte de la prévalence statistique concernant les MNA accueillis en France (majoritairement de sexe masculin, âgés de 16 ans et originaires d'Afrique de l'ouest). La représentation du MNA ainsi que certaines représentations liées à des scènes se déroulant dans le pays d'origine ont aussi été conçues en tenant compte de cette prévalence. Les tests de compréhension effectués permettent de valider les principes de conception visuelle retenus et notamment celui-ci: les MNA qui ne correspondent pas à la prévalence statique (Afghans par exemple), peuvent parvenir à «s'identifier» au MNA tel qu'il est représenté dans le livret.

\subsubsection{Stratégies de médiation : Se familiariser avec une utilisation multimodale}

50 Comme l'utilisation du livret par les MNA, sa prise en main par les professionnels n'est pas autonome. En effet, pour que l'ouverture d'un espace informationnel soit effectif, l'utilisation du livret nécessite d'être inscrite dans des stratégies de médiation multimodales (linguistiques et non-linguistiques). On parle de multimodalité pour intégrer l'ensemble des ressources (vocales et gestuelles, linguistiques et nonlinguistiques) qui ont un rôle dans la communication dans la production et l'interprétation du sens. L'objectif de ce guide d'utilisation vise ainsi à outiller les professionnels, afin qu'ils puissent étayer leurs stratégies de médiation pour transmettre une information effectivement adaptée. La familiarisation des professionnels avec l'utilisation multimodale du livret fait partie intégrante de l'outil non-linguistique proposé.

51 Pour chacune des planches, la rubrique " discours accompagnant la planche " propose une formulation, adaptée linguistiquement du contenu informationnel ainsi que des propositions de "stratégies de médiation » linguistiques et/ou multimodales, dans la rubrique du même nom. L'annexe 3 exemplifie la structure du guide d'utilisation pour la planche "Entretien d'évaluation 1 » qui donne un aperçu du fonctionnement de chacune des rubriques. Nous précisons que les adaptations du discours accompagnant les planches ainsi que les stratégies de médiation proposées dans ce guide sont largement inspirées des analyses menées sur les stratégies des professionnels accueillant les MNA que nous avons tenté de formaliser. 
52 Afin d'adapter le discours accompagnant les planches à la compréhension des MNA et donc favoriser l'accès au sens en limitant les incompréhensions, nous avons défini des principes d'adaptation linguistique: les constructions de phrases doivent être simplifiées, pour être plus proches des tournures de phrases utilisées à l'oral. Les MNA quand ils ont une compétence préalable en français, ou quand ils l'ont développée à leur arrivée en France, sont davantage familiers avec les structures de l'oral. Ainsi, par exemple, la formulation des questions évite l'inversion sujet-verbe qui est très peu fréquente à l'oral et peu familière aux jeunes. Nous avons par ailleurs privilégié les reprises nominales plutôt que les reprises pronominales (il, elle, la, le...) qui complexifient l'accès au sens et créent de l'ambiguïté. Certaines formulations pourront ainsi paraître insuffisamment académiques pour un lecteur locuteur natif de la langue française. Il s'agit d'un choix qui fait partie de notre démarche d'information adaptée : privilégiant l'accès au sens et non le français «normé » qui ne fait sens que pour un locuteur qui a une compétence en français écrit académique.

En outre, les stratégies de médiation que nous avons formalisées en réponse aux points de vigilance identifiés dans les données de terrain s s'articulent autour des catégories suivantes.

Des stratégies de reformulation : renforcer la compréhension des concepts clefs par des reformulations, des explicitations complémentaires.

- Par exemple : le concept d'isolement n'est pas forcément évident à appréhender pour les jeunes.

- Stratégie : expliciter davantage au moyen de reformulations et de pointages de l'index sur les représentations composant le concept d'isolement: "Quand tu es arrivé tu as dit que tu avais moins de 18 ans et que tu étais tout seul sur le territoire français, sans tes parents, sans ta famille, sans personne pour s'occuper de toi. Ici c'est toi. Ici ce sont tes parents. Toi tu es tout seul en France. Tes parents ne sont pas en France ".

Des stratégies de renforcement visuel : expliciter les représentations visuelles du livret, compléter ces explications par des gestes illustratifs, ou en lien avec la situation d'interaction immédiate.

- Par exemple: pour de multiples raisons (manque de connaissances et de bagage expérientiel), certains jeunes n'ont pas forcément l'habitude de se repérer grâce à une carte.

- Stratégie : effectuer un renforcement visuel, en pointant de l'index le pays d'origine du jeune sur la carte.

Des stratégies de convergence linguistique : utiliser un mot ou une formulation attestée dans les pratiques linguistiques des MNA permet de se rapprocher de l'univers linguistique des jeunes et de favoriser ainsi l'accès au sens par l'utilisation de mots signifiants pour eux.

- Par exemple : afin de faciliter l'accès au sens et de favoriser l'intercompréhension, il est parfois possible de converger vers le répertoire lexical du jeune en substituant des mots ou des formulations par des pratiques linguistiques qui lui sont plus habituelles et donc plus signifiantes pour lui.

- Stratégie: les jeunes en provenance d'Afrique de l'Ouest francophone utilisent régulièrement les expressions listées dans le discours accompagnant la planche pour parler de leurs difficultés morales/psychologiques («chauffer la tête ", "gâter tes nuits avec de mauvais songes »). Ils utilisent fréquemment le mot "gâter », pour parler de ce qui est endommagé, abimé, détérioré. Le mot «songe » peut être utilisé pour parler d'un rêve. Ils 
utilisent fréquemment l'expression "chauffer la tête ", pour parler de leurs difficultés, de leurs problèmes, de leurs tourments, de leurs maux. Ils peuvent également dire que la situation les « fatigue ».

Des stratégies d'adaptation socio-culturelle : adapter le discours en fonction du bagage socio-culturel du jeune, des éléments expérientiels qui peuvent faire sens pour lui.

- Par exemple : afin de favoriser l'accès au sens et l'intercompréhension, il peut être utile d'adapter le discours selon la zone géographique de provenance du jeune pour utiliser des références plus signifiantes pour lui.

- Stratégie : citer différents groupes terroristes selon la zone concernée : Afrique de l'ouest (Boko Haram, Ansar Dine, Aqmi, Katiba Macina...) ; Moyen Orient (Daech, Al-Qaïda (dans la péninsule arabique), Ansar Beït al-Maqdess, Front al-Nosra...), Asie/Afghanistan (Talibans, Abou Sayyaf, Al-Quaïda, Jemaah Islamiyah...).

Précisons que ces stratégies de médiation multimodales visent également, au-delà de la transmission d'information, et dans le but de favoriser l'appropriation par le jeune, de permettre la familiarisation avec certains concepts circulants dans l'environnement institutionnel français des MNA, notamment par des stratégies de reformulations linguistiques et gestuelles, ainsi que visuelles (pointage sur le livret ou dans la situation d'interaction immédiate) favorisant l'association mot-sens.

\section{Perspectives conclusives}

Le livret et son guide d'utilisation ont été diffusés sur le site du projet MIMNA (2019) en octobre. Les retours des structures de terrain ainsi que des professionnels nous confortent dans la pertinence de notre démarche d'information adaptée aux MNA.

Les besoins de formation des professionnels pour la prise en main du livret MIMNA, ainsi que les besoins en accompagnement pour l'élaboration de leurs propres outils de médiation, sur la base du livret MIMNA, sont conséquents. Ces besoins de terrain nous poussent à concevoir l'élaboration d'autres ressources permettant de mettre en scène la multimodalité (vidéo notamment).

Une des perspectives de poursuite du projet vise également à évaluer l'impact de ce livret sur l'accès à l'information des MNA. Si les tests de compréhension, que nous avons réalisés durant la phase de conception du livret, nous permettent de présager d'un impact certain, nous visons à formaliser davantage cette phase d'évaluation auprès des professionnels et des MNA d'autres structures. Ceci nous permettra de pouvoir évaluer les réajustements nécessaires.

\section{BIBLIOGRAPHIE}

ANESM (2017). Recommandations de bonnes pratiques professionnelles : L'accompagnement des mineurs non accompagnés dits « mineurs isolés étrangers ». 
Bordon, E. (2004). L'interprétation des pictogrammes. Paris : L'Harmattan.

Conseil de l'Europe (2018). How to convey child-friendly information in migration. A handbook for frontline professionnals.

Conseil de l'Europe. (2019). Promoting child-friendly approaches in the area of migration.

Conseil de l'Europe. (2009). Assurer la pleine participation grâce à la conception universelle.

Coron, G. (2018). Bonnes pratiques professionnelles dans l'accueil et la prise en charge des mineurs non accompagnés : L'importance de respecter l'intérêt supérieur de l'enfant. Jeunes et Mineurs en Mobilité, 4, 8-17.

Eco, U. (1970). Sémiologie des messages visuels. Communications, (15), 11-51.

Estève, I., Coron, G. \& Gaultier, S. (à paraître, 2020). Accueillir et accompagner les mineurs non accompagnés en France : Appréhender les problématiques d'accès à l'information pour optimiser les outils de médiation. CIICAR : International Conference Cross-Linguistic and Cross-Cultural Communication for Refugees and Asylum Seekers. Séville : Comares.

Estève, I. \& Coron, G. (à paraître, 2020). Apports de la (socio)linguistique appliquée pour la Médiation de l'Information à destination des MNA en France. Étude en linguistique appliquée.

Gardiès, C. et Rinaudo, J-L. (2015). « Éditorial », Distances et médiations des savoirs, 12 [en ligne : http://journals.openedition.org/dms/1174]

Grosjean, F. (1984). Le bilinguisme : Vivre avec deux langues. Revue Tranel (Travaux neuchâtelois de linguistique), 7, 15-41.

Jewitt, C. \& Kress, G. (2010). Multimodality, literacy and school English. The Routledge international handbook of English, language and literacy teaching. London/New York : Routledge.

Kerbrat-Orecchioni, C. (1986). L'implicite. Paris : Armand Colin.

Kerbrat-Orecchioni, C. (2005). Le discours en interaction. Paris : Armand Colin.

Kress, G. (2019). L’apprentissage en tant que travail sémiotique : Vers une pédagogie de la reconnaissance. In V. Rivière et $\mathrm{N}$. Blanc (Éds), Observer la multimodalité en situation éducative (pp. 23-48). Lyon : ENS Éditions.

Laforest, M. \& Vincent, D. (1999). Incompréhension et malentendu : Deux manifestations de la coconstruction du sens, Langues et linguistique, (25), 111-144.

Liquète, V. (2015). L'accessibilité web comme porte et enjeu de médiation des savoirs, Distances et médiations des savoirs, (12). http://journals.openedition.org/dms/1200

Marandon, G. (2003). Au-delà de l'empathie, cultiver la confiance : Clés pour la rencontre interculturelle. Revista CIDOB d'afers internacionals, 259-282.

Mazur-Palandre, A. \& Colón de Carvajal, I. (2019). Conclusion. La multimodalité du langage : Un domaine encore et toujours à explorer. In A. Mazur-Palandre et I. Colon de Carvajal. (Éds.), Approches plurielles de la multimodalité du langage (pp. 317-332). Grenoble : UGA Éditions.

Médiation de l'information pour les mineurs non accompagnés (MIMNA). (2019). Livret MIMNA. https://mimna.univ-grenoble-alpes.fr/

Mottez, B. ([1985] 2006). Les sourds existent-ils ? Paris : L'Harmattan.

OCDE (2000). La littératie à l'ère de l'information, Rapport final de l'enquête internationale sur la littératie des adultes. Paris : France.

Paveau, M. A. (2006). Les pré-discours : Sens, mémoire, cognition. Paris : Presses Sorbonne nouvelle. 
Ruel J. \& Allaire, C. (Éds). (2018). Communiquer pour tous : Guide pour une information accessible. Saint-Maurice : Santé publique France.

Tourneux, H. (1993). La perception des pictogrammes phytosanitaires par les paysans du NordCameroun. Coton et fibres tropicales, 48(1), 41-48.

Vallerie, B. \& Le Bossé, Y. (2006). Le développement du pouvoir d'agir (empowerment) des personnes et des collectivités : De son expérimentation à son enseignement. Les Sciences de l'éducation - Pour l'Ère nouvelle, 39(3), 87-100.

Vervliet, M., Lammertyn, J., Broekaert, E. \& Derluyn, I. (2014). Longitudinal follow-up of the mental health of unaccompanied refugee minors. Eur Child Adolesc Psychiatry, (23), 337-346.

Vetier, T. (2016). Les mineurs isolés étrangers pris en charge par la protection de l'enfance en France : Jeunes « à intégrer » entre mobilités multiples et immobilisation institutionnelle. Cahiers internationaux de sociolinguistique, 1 (9), 291-316.

\section{ANNEXES}

\section{Annexes}

Annexe 1 : Aperçu de la planche « Arrivée »

La planche Arrivée vise à indiquer au jeune où il se trouve en France et la raison de sa prise en charge sur la base de sa déclaration de minorité/isolement.
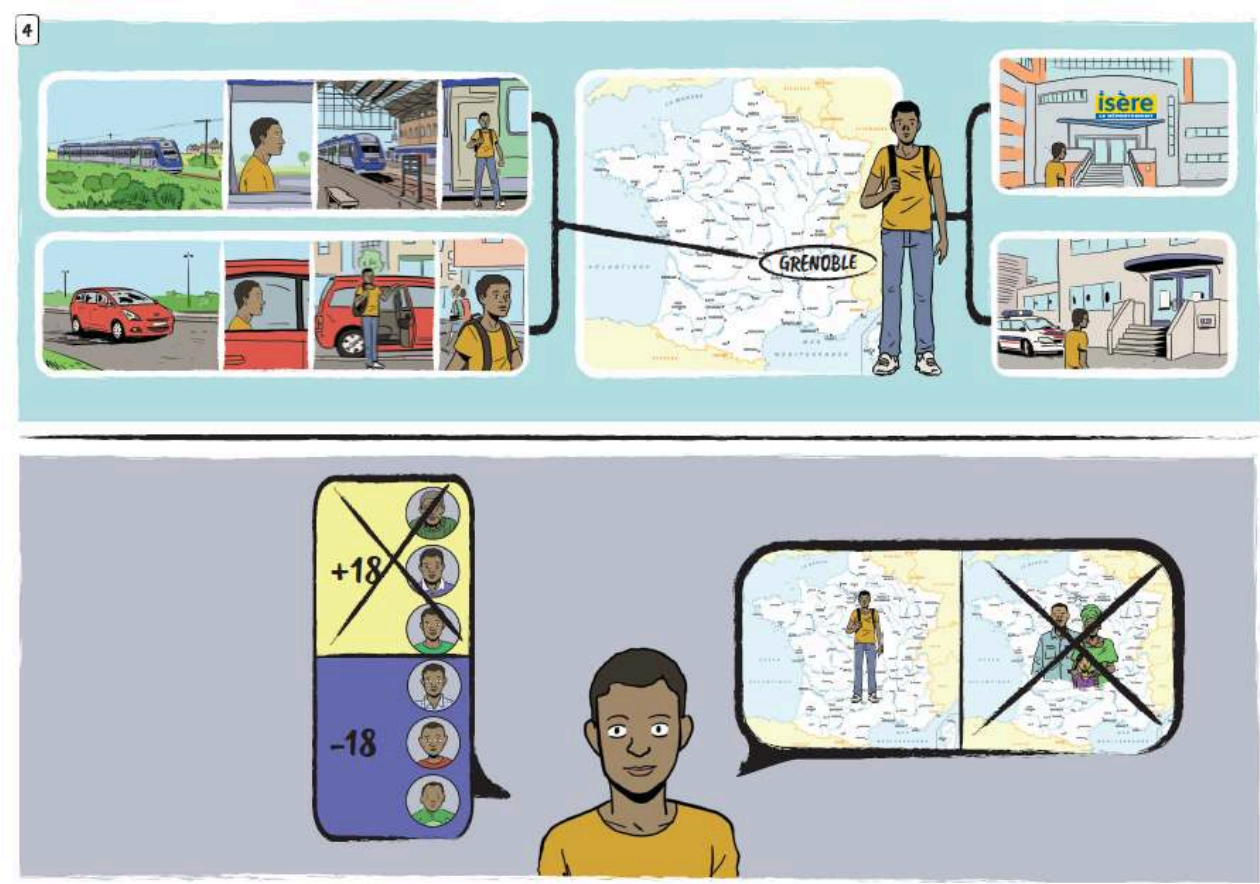

Annexe 2 : Aperçu de la planche « Hébergement »

La planche hébergement vise à expliquer la procédure de mise à l'abri, la prise en charge par un service d'accueil d'urgence de protection de l'enfance et les modalités d'hébergement. 

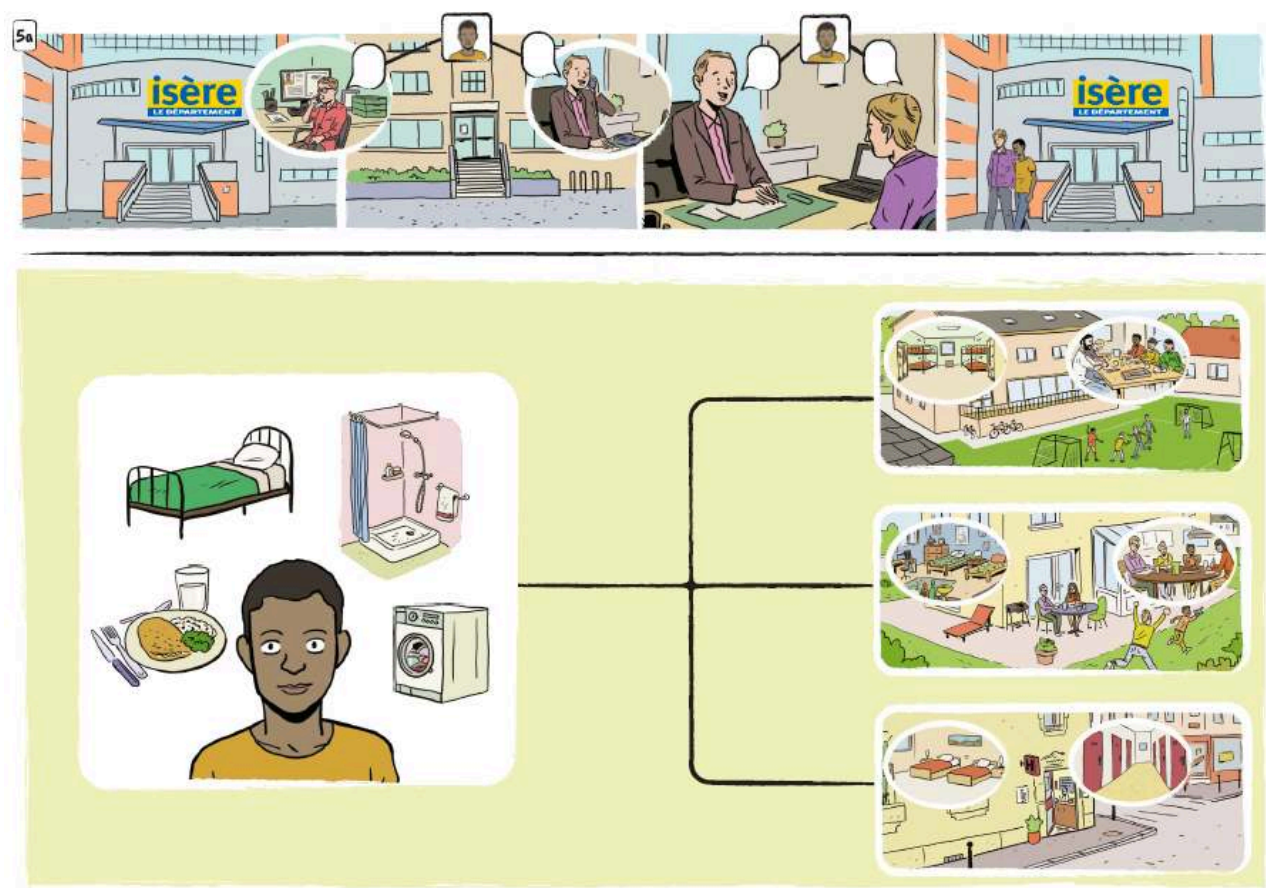

Annexe 3 : Aperçu du guide d'utilisation pour la planche « Entretien évaluation 1 » 


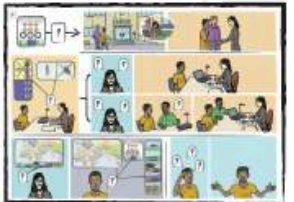

- Objectifs informationnels -

- Expliquer lentretien dévaluation sociale de minorité-isolement, ses objectifs, son déroulement et ses enjeux

Informer le jeune de son droit à recourit a un interprete pour cet entretien.

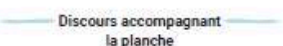
la planche

Dans quelques jours, on ne sait pas quand mais on te dira quand on saura, tu vas devoir aller au Departement, au Conseil Departemental pour un rendezwous, un Tuvas rencontrec quelayinn une personne. une professionnelle que ron appelle une evaluatrice. Tu vas denoir parler avec révaluatrice, répondre à ses questions.

Elle va te poser des questions pour savoir si tues bien mineur, situ as bien moins do 18 ans sans tes parents, sans etre accompa

Si tu comprends bien le francais, si tu parles bien le français, tu seras seul avec

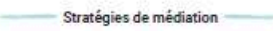

- Les concepts de minorité et disolement ne sont pas forcement évidents apprenender pour les jeunes

être nécessai: de consacrer une nowvelle fois du tempg visuelles renvoyantàdes notions complexes mais pourtant essentelles. Pour cel precisons que la representation visue ou principe de minorite/majome consider le même personnage a differents ages de la barte des 18 ans le personnage a les mêmes caractéristiques physiques. Lisolement peut être expliqué au moyen of reformulations et de pointages de linder sur les representations composant as oit que tuavais moing de 18 ans es que th étais tout seul surle termitore francais sants tes parents, sans ta familie, sans personn pour sbcouper de toi. fici cest toilicice son es parents. Toi tues tout seu/ en Friance. Tes

L Le rolle d'un intercrète n'est pas toupurs facile à appréhender pour les jeunes. $\rightarrow$ Stratégie : II peut donc converi dexpliquer quels sont le role et las fonction dun interprete comme par exemple : o U

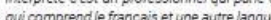
souvent la langue du pays dorigine de sees parents ou du pays où î̉est né Par exemple je connais un interprete quil sappelile (nom de Finterprete) qui vis en France depuis très longtemps, in parle tres bien fiançais, mais et il parle tres bien (langue(s) du pays de naissance) Lintercrete est payé pourtradule es questions et les reponses des personn qui ne parlent pas la méme langue pendant in rendez-vous importants,

evaluatrice pendant lentretien.

Sif tu ne comprends pas bien le francais. ou que tu ne parles pas bien le français, il aura un interprete, dans une langue que tu traduira les questions de lévaluatrice et tes

Lévaluatrice va te poser un peu les mèmes questions que moi aujourd hui, maintenant. mais tu devras répondre avec beaucoup
plus de détalis. Tu devras être précis.

Par exemple, révaluatrice va te poser des questions surton voyage, sur les transports, France, les pays que tu as traversé depuis que tu as quitte (nom du pays d'origine du jeune) avant dentrer en France. II faudra donc que tu dises par ou tu es passé, par quels pajs. Tu dens dire combien de cemps tu as passe dans chaque ville et duré. Tu devras dice les transports que tu as utilisé. Si tu es voyagé en avion en train en voiture, en bateau, à pied

I| faudra trte très précis dans tes réponses faudra que tu dises, que tu donnes les informations dont tu te rappelles, dont 1 te souviens. Si tu ne sais pas ou que tu ne te souviens pas, tu peux dire r je ne sals pas ou bien . je ne me souviens pas, , ll ne faut pas inventer les réponses si tu ne connais pas les reponses. Si tu ne comprends pas la prends pas

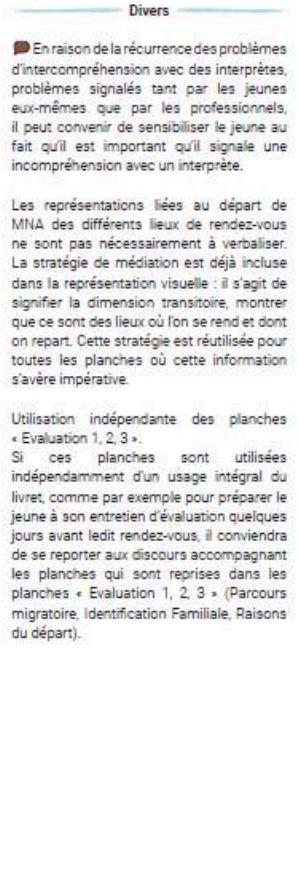

\section{NOTES}

1. Comme le révèle cet extrait d'entretien, le livret a permis aux professionnels d'ouvrir, autour de cet outil de médiation, un espace informationnel avec les MNA non francophones jusqu'alors inexistant du fait d'une incompréhension réciproque : «en fait au départ/ avant qu'il y ait ce livret, 
on s'est rendu compte que quand les jeunes ne parlaient pas français ou pas suffisamment, on les recevait pas/ parce qu'on était dans un face-à-face sans aucune médiation/ sans aucun support de médiation qui faisait que euh... on était euh... démunis puisqu'on était sur du purement verbal/ on les reçoit tous maintenant, ce qui veut dire que ils ont tous un temps qui leur est consacré et ça c'est quand même extrêmement important/ mais quoiqu'ils en comprennent/ et nous on n'est pas en difficulté même si ils comprennent rien parce qu'on a cette médiation-là/ donc nous on se cramponne, on s'accroche à ça et donc on peut supporter l'incompréhension/ et on peut même composer devenir créatifs imaginatifs je ne sais quoi autour de l'incompréhension parce qu'on a le support » [En-reco-CS1, Généralités]

2. «L'accessibilité revient à penser un schéma complexe et multidimensionnel de la médiation (Liquète, 2010), où l'on tente de rapprocher progressivement les logiques des producteurs d'information, des professionnels de l'information et des usagers, en considérant pour ces derniers, non seulement leurs besoins d'information, mais également leurs expériences et la diversité de leurs contraintes. » (Liquète, 2015)

3. Nous rejoignons la démarche de médiation développée par Fabre et Gardiès : « Si l'information est considérée comme un contenu cognitif situé dans un processus de communication où le récepteur a un rôle primordial d'activation, d'interprétation et d'appropriation, « les nouveaux processus de médiation doivent [..] prendre en compte l'activation du sens par le récepteur [...], pour lui permettre d'appréhender et s'approprier l'information afin de construire des connaissances signifiantes pour lui » (Fabre \& Gardiès, 2010 cités par Gardiès \& Rinaudo, 2015).

4. Les données sur lesquelles nous nous appuyons dans cette partie sont issues du rapport annuel d'activité de la mission MNA du Ministère de la Justice (2018), ainsi que sur une enquête de terrain, en l'absence de données officielles concernant les profils linguistiques, scolaires des MNA. Une étude quantifiée a été effectuée sur la base de la totalité des élèves scolarisés (N=194 élèves) dans le dispositif de scolarité interne du Charmeyran.

5. Précisons que $84 \%$ des MNA qui arrivent en France sont âgés de 16 ans ou moins.

6. $67 \%$ des MNA proviennent d'Afrique de l'Ouest (31\% de Guinée, $20 \%$ du Mali et $16 \%$ de Côte d'Ivoire) et pour $33 \%$ d'autres pays.

7. La majorité d'entre eux est allophone : la langue française n'est pas leur langue première, mais plus souvent, pour ceux qui ont une compétence dans cette langue à leur arrivée, une langue de scolarisation ou une lingua franca, langue véhiculaire, c'est-à-dire une langue utilisée pour les échanges quotidiens ne faisant pas forcément l'objet d'un apprentissage formel.

8. La majorité d'entre eux présentent donc de faibles compétences en littératie: «Aptitude à comprendre et à utiliser l'information écrite dans la vie courante, à la maison, au travail et dans la collectivité en vue d'atteindre des buts personnels et d'étendre ses connaissances et ses capacités. » (OCDE, 2000)

9. Leur bagage scolaire est assez faible: $40 \%$ d'entre eux n'ont pas été ou peu scolarisés antérieurement, $25 \%$ ont atteint un niveau primaire et $35 \%$ un niveau collège.

10. Leurs connaissances socio-culturelles de la France sont souvent erronées, ou tout au moins partielles et parfois stéréotypées, façonnées tout autant par les représentations circulantes dans leur pays d'origine que par l'intermédiaire des autres migrants et d'autres acteurs (passeurs, intermédiaires, professionnels, volontaires) rencontrés durant leurs parcours migratoires (Coron, à paraître 2019).

11. Nous empruntons ce concept à Mottez ([1985] 2006) développé pour rendre compte du contexte de la surdité.

12. «La notion d'“empowerment" [...] réfère à "la possibilité pour les personnes ou les communautés de mieux contrôler leur vie" (Rapport, 1987), ou, de façon plus spécifique, à un processus caractérisé par l'exercice d'une plus grande maîtrise sur l'atteinte d'objectifs importants pour une personne, une organisation ou une communauté.» (Vallerie \& Le Bossé, 2006, p. 88) 
13. «Les pré-discours sont en effet des opérateurs dans la négociation du partage, de la transmission et de la circulation du sens dans les groupes sociaux. Je les définis comme un ensemble de cadres prédiscursifs collectifs qui ont un rôle instructionnel pour la production et l'interprétation du sens en discours. J'entends par pré-discours des contenus sémantiques (au sens large de culturel, idéologique, encyclopédique), c'est-à-dire des savoirs, des croyances et des pratiques ». (Paveau, 2006, p. 7)

14. En effet, les MNA proviennent souvent de pays plurilingues. Si ces langues font partie de leurs répertoires langagiers, ils maîtrisent ces langues à des degrés divers et dans des domaines différents de la vie quotidienne. Le bilinguisme est ainsi très souvent fonctionnel. La langue choisie pour la traduction ne correspond pas toujours à la langue première du jeune, et n'est donc pas nécessairement celle qu'il maitrise le plus. En outre, quand bien même dans certaines situations d'interprétation, la langue est bien la langue première du jeune, le vocabulaire administratif, médical, social, etc. utilisé par le professionnel (et traduit par l'interprète) n'est pas nécessairement connu du jeune. En effet, la compétence développée dans une langue correspond aux besoins quotidiens de communication qu'on en a (Grosjean, 1984).

15. Pour une analyse plus fine de ces résultats, nous renvoyons à ce que nous avons développé par ailleurs (Estève, Coron \& Gaultier, à paraître, 2020 ; Estève \& Coron, à paraître 2020).

16. "Nous parlerons : a) d'incompréhensions lorsqu'il y a impossibilité totale ou partielle, pour un auditeur, d'interpréter un segment plus ou moins vaste de discours, c'est-à-dire de lui attribuer un sens ; b) de malentendu lorsqu'il y a interprétation divergente d'un même segment de discours par les interlocuteurs, mais ce sens n'est pas celui que cherchait à communiquer le locuteur. » (Laforest \& Vincent, 1999, p. 116)

17. «L'énoncé a donc le sens ou plutôt les sens que les participants à l'interaction lui prêtent, en fonction de leurs compétences différentes et de nature diverse : “Un énoncé n'a pas de sens-ensoi." » (Kerbrat-Orecchioni, 1986, p. 309)

18. «Le sens des énoncés n'est pas, en conversation spontanée, donné, fixé une fois pour toutes, [...] les interlocuteurs discutent, révisent, négocient l'interprétation accordée ou à accorder au discours. [...] L'activité conversationnelle ne doit pas être vue comme l'alternance de la parole entre interlocuteurs tantôt actifs (lorsqu'ils sont locuteurs), tantôt passifs (lorsqu'ils sont auditeurs), mais plutôt comme une activité interactionnelle qui demande la coopération simultanée des participants, qu'ils soient locuteurs ou auditeurs. La conversation se caractérise donc par la succession d'actions de co-construction du sens. » (Laforest \& Vincent, 1999, p. 113).

19. La « conception universelle» est sous-tendue par l'idée que la prise en compte des besoins spécifiques dès la conception servira à d'autres besoins ponctuels ou plus ou moins durables. La rampe d'accès à un bâtiment est un exemple assez parlant de la prise en compte de besoins spécifiques (besoins permanents des publics à mobilité réduite) dès la conception qui peuvent par ailleurs servir les besoins d'accessibilité ponctuels plus ou moins durables d'autres usagers : de la personne âgée en déambulateur, de la personne amenée à se déplacer en béquille, des parents avec une poussette, de l'usager avec une valise, etc.

20. Loi n 2002-2 du 2 janvier 2002 rénovant l'action sociale et médico-sociale.

21. Nous entendons par scénarisation de l'information, un principe de représentation utilisé pour la bande dessinée. 


\section{RÉSUMÉS}

Nous rendrons compte de la démarche de conception du livret d'accueil visuel et de son guide d'utilisation que nous avons élaborés dans le cadre du projet Médiation de l'information pour les mineurs non accompagnés (MIMNA). Entrecroiser l'expertise des professionnels travaillant auprès des MNA, et une approche sociolinguistique des problématiques de médiation a permis de formaliser des principes et des stratégies de médiation, sur la base des données de terrain, afin d'élaborer ces outils de médiation dans une démarche d'information effectivement adaptée aux MNA. Nous entendons par information adaptée le fait qu'elle permette au MNA, non seulement d'activer du sens mais de s'approprier l'information et de construire des connaissances qui sont signifiantes pour lui.

We will present the design process of the visual welcome booklet and its user guide that we conceived in the MIMNA project (Mediation of Information for Unaccompanied Minors). Crossing the expertise of professionals working with unaccompanied minors and a sociolinguistic approach to mediation issues led us formalise mediation principles and strategies, based on field data. These mediations tools have been developed as part of an information approach effectively adapted to Unacompanied Minors. We understand by adapted information: it allows the Unacompagnied Minors not only to activate meaning but also to appropriate the information and to build knowledge that is meaningful to him/her.

\section{INDEX}

Mots-clés : Mineurs non accompagnés (MNA), médiation de l'information, conception universelle, information adaptée, sociolinguistique

Keywords : Unacompagnied Minors, Mediation of Information, Universal design, sociolinguistics

\section{AUTEUR}

\section{ISABELLE ESTÈVE}

Maître de conférences, Laboratoire Lidilem, Université Grenoble-Alpes 